\title{
New oscillation results for second-order neutral delay dynamic equations
}

\author{
Chenghui Zhang ${ }^{1}$, Ravi P Agarwal ${ }^{2}$, Martin Bohner ${ }^{3 *}$ and Tongxing $\mathrm{Li}^{1}$
}

\footnotetext{
"Correspondence: bohner@mst.edu ${ }^{3}$ Department of Mathematics and Statistics, Missouri S\&T, Rolla, MO 65409-0020, USA

Full list of author information is available at the end of the article
}

\begin{abstract}
This paper is concerned with oscillatory behavior of a certain class of second-order neutral delay dynamic equations

$$
\left(r(t)[x(t)+p(t) x(\tau(t))]^{\Delta}\right)^{\Delta}+q(t) x(\delta(t))=0,
$$

on a time scale $\mathbb{T}$ with sup $\mathbb{T}=\infty$, where $0 \leq p(t) \leq p_{0}<\infty$. Some new results are presented that not only complement and improve those related results in the literature, but also improve some known results for a second-order delay dynamic equation without a neutral term. Further, the main results improve some related results for second-order neutral differential equations.
\end{abstract}

MSC: 34K11; 34N05; 39A 10

Keywords: oscillation; neutral delay dynamic equation; second-order equation; time scale

\section{Introduction}

In this paper, we are concerned with oscillation of a class of second-order neutral delay dynamic equations,

$$
\left(r(t)[x(t)+p(t) x(\tau(t))]^{\Delta}\right)^{\Delta}+q(t) x(\delta(t))=0,
$$

where $t \in\left[t_{0}, \infty\right)_{\mathbb{T}}:=\left[t_{0}, \infty\right) \cap \mathbb{T}$, and

$\left(H_{1}\right) r, p, q \in \mathrm{C}_{\mathrm{rd}}\left(\left[t_{0}, \infty\right)_{\mathbb{T}}, \mathbb{R}\right), r(t)>0,0 \leq p(t) \leq p_{0}<\infty, q(t)>0 ;$

$\left(H_{2}\right) \delta \in \mathrm{C}_{\mathrm{rd}}\left(\left[t_{0}, \infty\right)_{\mathbb{T}}, \mathbb{T}\right), \delta(t) \leq t, \lim _{t \rightarrow \infty} \delta(t)=\infty, \tau \circ \delta=\delta \circ \tau$;

$\left(H_{3}\right) \tau \in C_{\mathrm{rd}}^{1}\left(\left[t_{0}, \infty\right)_{\mathbb{T}}, \mathbb{T}\right), \tau(t) \leq t, \tau^{\Delta}(t) \geq \tau_{0}>0, \tau\left(\left[t_{0}, \infty\right)_{\mathbb{T}}\right)=\left[\tau\left(t_{0}\right), \infty\right)_{\mathbb{T}}$, where $\tau_{0}$ is a constant.

Throughout this paper, we assume that solutions of (1.1) exist for any $t \in\left[t_{0}, \infty\right)_{\mathbb{T}}$. A solution $x$ of (1.1) is called oscillatory if it is neither eventually positive nor eventually negative; otherwise, we call it nonoscillatory. Equation (1.1) is said to be oscillatory if all its solutions oscillate.

A time scale $\mathbb{T}$ is an arbitrary nonempty closed subset of the real numbers $\mathbb{R}$. Since we are interested in oscillatory behavior, we suppose that the time scale under consideration is not bounded above and is a time scale interval of the form $\left[t_{0}, \infty\right)_{\mathbb{T}}$. For some concepts related to the notion of time scales, see $[1,2]$.

(C) 2012 Zhang et al.; licensee Springer. This is an Open Access article distributed under the terms of the Creative Commons Attribution License (http://creativecommons.org/licenses/by/2.0), which permits unrestricted use, distribution, and reproduction in any medium, provided the original work is properly cited. 
Recently, there has been an increasing interest in obtaining sufficient conditions for oscillatory or nonoscillatory behavior of different classes of differential equations and dynamic equations on time scales; we refer the reader to the papers [3-38]. In the following, we present some details that motivate the contents of this paper. Regarding oscillation of second-order neutral differential equations, Grammatikopoulos et al. [16] established that the condition

$$
\int_{t_{0}}^{\infty} q(s)[1-p(s-\delta)] \mathrm{d} s=\infty
$$

ensures oscillation of the linear neutral differential equation

$$
(x(t)+p(t) x(t-\tau))^{\prime \prime}+q(t) x(t-\delta)=0 .
$$

Later, Grace and Lalli [15] obtained that the conditions

$$
\int_{t_{0}}^{\infty} \frac{\mathrm{d} t}{r(t)}=\infty
$$

and

$$
\int_{t_{0}}^{\infty}\left(\theta(s) q(s)[1-p(s-\delta)]-\frac{\left(\theta^{\prime}(s)\right)^{2} r(s-\delta)}{4 \theta(s)}\right) \mathrm{d} s=\infty
$$

for some positive function $\theta \in C^{1}\left(\left[t_{0}, \infty\right), \mathbb{R}\right)$ ensure oscillation of the linear neutral differential equation

$$
\left(r(t)(x(t)+p(t) x(t-\tau))^{\prime}\right)^{\prime}+q(t) x(t-\delta)=0 .
$$

Baculíková and Džurina [8] established that the conditions

$$
1<p_{1} \leq p(t) \leq p_{2}<\infty, \quad \tau^{\prime}(t) \geq \tau_{0}>0, \quad \tau \circ \delta=\delta \circ \tau, \quad \delta(t)<\tau(t)<t,
$$

and

$$
\int_{t_{0}}^{\infty} q(s) \mathrm{d} s=\infty
$$

ensure oscillation of the linear neutral differential equation

$$
\left(r(t)[x(t)+p(t) x(\tau(t))]^{\prime}\right)^{\prime}+q(t) x(\delta(t))=0 .
$$

Recently, Zhong et al. [38] improved this result, and they obtained that the conditions (1.2), $p \geq 0, p \neq 1$, and

$$
\int_{t_{0}}^{\infty}\left(\theta(s) q(s) \frac{1-\varepsilon}{1+p(1+\varepsilon)}-\frac{\left(\theta^{\prime}(s)\right)^{2} r(\delta(s))}{4 \theta(s) \delta^{\prime}(s)}\right) \mathrm{d} s=\infty
$$


for some constant $\varepsilon \in(0,1)$ and for some positive function $\theta \in C^{1}\left(\left[t_{0}, \infty\right), \mathbb{R}\right)$ guarantee oscillation of the linear neutral differential equation

$$
\left(r(t)(x(t)+p x(t-\tau))^{\prime}\right)^{\prime}+q(t) x(\delta(t))=0 .
$$

Hasanbulli and Rogovchenko [20] used the standard integral averaging technique to obtain some new oscillation criteria for the second-order neutral delay differential equation

$$
\left(r(t)(x(t)+p(t) x(t-\tau))^{\prime}\right)^{\prime}+q(t) f(x(t), x(\sigma(t)))=0 .
$$

For oscillation of second-order dynamic equations on time scales, Erbe et al. [13] established a sufficient condition which ensures that the solution $x$ of the delay dynamic equation

$$
\left(r(t) x^{\Delta}(t)\right)^{\Delta}+q(t) x(\tau(t))=0
$$

is either oscillatory or satisfies $\lim _{t \rightarrow \infty} x(t)=0$ under the condition

$$
\int_{t_{0}}^{\infty} \frac{1}{r(t)} \int_{t_{0}}^{t} q(s) \Delta s \Delta t=\infty
$$

Zhang [36] obtained some oscillation results for (1.4) in the case where

$$
\int_{t_{0}}^{\infty} \frac{1}{r(t)} \int_{t_{0}}^{t} q(s) \int_{s}^{\infty} \frac{\Delta u}{r(u)} \Delta s \Delta t=\infty
$$

Agarwal et al. [4], Saker [29], and Tripathy [33] considered the equation

$$
\left(r(t)(x(t)+p(t) x(t-\tau))^{\Delta}\right)^{\Delta}+q(t) x(t-\delta)=0,
$$

and established some oscillation results for (1.6) provided that $0 \leq p(t) \leq 1$ and

$$
\int_{t_{0}}^{\infty} \frac{\Delta t}{r(t)}=\infty
$$

In particular, Tripathy [33] obtained some oscillation criteria for (1.6) when (1.7) holds and $0 \leq p(t) \leq p_{0}<\infty$, and established that the condition

$$
\int_{t_{0}}^{\infty} \min \{q(s), q(s-\tau)\} \Delta s=\infty
$$

ensures oscillation of (1.6).

The question regarding the study of oscillatory properties of (1.1) (including the case when $\mathbb{T}=\mathbb{R})$ has been solved by some recent papers; see $[4,8,17,19,25,29,32,33]$ etc. Based on the conditions $\tau(t) \leq t$ and $\sigma(t) \leq t$, they established some results. The ideas can be divided into two aspects, i.e., comparison methods and the Riccati transformation. In order to compare our results in Section 2 with those related subjects in $[4,8,17,19,25$, $29,32,33]$, we list their results as follows. 
Theorem 1.1 (See [8]) Let (1.2) hold and $\tau^{-1}$ be the inverse function of $\tau$. Assume $\left(H_{1}\right)-\left(H_{3}\right)$ for $\mathbb{T}=\mathbb{R}$ and $\delta(t) \leq \tau(t) \leq t$. If

$$
\liminf _{t \rightarrow \infty} \int_{\tau^{-1}(\delta(t))}^{t}\left(\min \{q(s), q(\tau(s))\} \int_{t_{0}}^{\delta(s)} \frac{\mathrm{d} u}{r(u)}\right) \mathrm{d} s>\frac{\tau_{0}+p_{0}}{\tau_{0} \mathrm{e}}
$$

then (1.3) is oscillatory.

Theorem 1.2 (See $[17,32])$ Let $(1.2)$ hold. Assume $\left(H_{1}\right)-\left(H_{3}\right)$ for $\mathbb{T}=\mathbb{R}$ and $t \geq \delta(t) \geq \tau(t)$. If there exists a positive function $\alpha \in \mathrm{C}^{1}\left(\left[t_{0}, \infty\right), \mathbb{R}\right)$ such that

$$
\limsup _{t \rightarrow \infty} \int_{t_{0}}^{t}\left[\alpha(s) \min \{q(s), q(\tau(s))\}-\left(1+\frac{p_{0}}{\tau_{0}}\right) \frac{r(\tau(s))\left(\alpha^{\prime}(s)\right)^{2}}{4 \tau_{0} \alpha(s)}\right] \mathrm{d} s=\infty
$$

then (1.3) is oscillatory.

Theorem 1.3 (See $[4,29])$ Let (1.7) hold. Assume $\left(H_{1}\right)-\left(H_{3}\right)$ for $\tau(t)=t-\tau, \delta(t)=t-\delta$, and $p_{0}=1$. If there exists a positive function $\alpha \in C_{\mathrm{rd}}^{1}\left(\left[t_{0}, \infty\right)_{\mathbb{T}}, \mathbb{R}\right)$ such that

$$
\limsup _{t \rightarrow \infty} \int_{t_{0}}^{t}\left[\alpha(s) q(s)(1-p(s-\delta))-\frac{r(s-\delta)\left(\alpha^{\Delta}(s)\right)^{2}}{4 \alpha(s)}\right] \Delta s=\infty
$$

then (1.1) is oscillatory.

Theorem 1.4 (See $[19,25])$ Let $(1.7)$ hold. Assume $\left(H_{1}\right)-\left(H_{3}\right)$ and $t \geq \delta(t) \geq \tau(t)$. If there exists a positive function $\alpha \in \mathrm{C}_{\mathrm{rd}}^{1}\left(\left[t_{0}, \infty\right)_{\mathbb{T}}, \mathbb{R}\right)$ such that

$$
\limsup _{t \rightarrow \infty} \int_{t_{0}}^{t}\left[\alpha(s) \min \{q(s), q(\tau(s))\}-\left(1+\frac{p_{0}}{\tau_{0}}\right) \frac{r(\tau(s))\left(\alpha^{\Delta}(s)\right)^{2}}{4 \tau_{0} \alpha(s)}\right] \Delta s=\infty,
$$

then (1.1) is oscillatory.

Theorem 1.5 (See [33]) Let (1.7) hold. Assume $\left(H_{1}\right)-\left(H_{3}\right)$ for $\tau(t)=t-\tau, \delta(t)=t-\delta$, and $\delta \geq \tau>0$. If there exists a positive function $\alpha \in \mathrm{C}_{\mathrm{rd}}^{1}\left(\left[t_{0}, \infty\right)_{\mathbb{T}}, \mathbb{R}\right)$ such that

$$
\limsup _{t \rightarrow \infty} \int_{t_{0}}^{t}\left[\alpha(s) \min \{q(s), q(s-\tau)\}-\frac{\left(1+p_{0}\right) r(s-\tau)\left(\alpha^{\Delta}(s)\right)^{2}}{4 \alpha(s)}\right] \Delta s=\infty,
$$

then (1.1) is oscillatory.

The natural question now is: Can one obtain new oscillation criteria for (1.1) that improve the results in $[4,19,25,29,33]$ ? The aim of this paper is to give an affirmative answer to this question. As a special case when $\mathbb{T}=\mathbb{R}$, the obtained results improve those by $[8,15$, $17,32,38]$. As a special case when $p(t)=0$, the obtained results improve those reported in $[13,36]$.

\section{Main results}

In this section, we establish the main results. All functional inequalities considered in this section are assumed to hold eventually, that is, they are satisfied for all $t$ large enough. For 
our further references, let us denote

$$
Q(t):=\min \{q(t), q(\tau(t))\} \quad \text { and } \quad z(t):=x(t)+p(t) x(\tau(t)) .
$$

Theorem 2.1 Assume $\left(H_{1}\right)-\left(H_{3}\right)$ and (1.7). If there exist functions $\eta, a \in \mathrm{C}_{\mathrm{rd}}^{1}\left(\left[t_{0}, \infty\right)_{\mathbb{T}}, \mathbb{R}\right)$ such that $\eta(t)>0, a(t) \geq 0$, and

$$
\limsup _{t \rightarrow \infty} \int_{t_{2}}^{t}\left(\eta^{\sigma}(s) Q(s) \frac{\int_{t_{1}(s)}^{\delta(v)} \frac{\Delta v}{r(v)}}{\int_{t_{1}}^{\sigma(s)} \frac{\Delta v}{r(v)}}-E(s)\right) \Delta s=\infty
$$

for all sufficiently large $t_{1}$ and for some $t_{2} \geq t_{1}$, where

$$
\begin{aligned}
E(s):= & -\eta^{\sigma}(s)\left[r(s) a^{2}(s) \frac{\int_{t_{1}}^{s} \frac{\Delta v}{r(v)}}{\int_{t_{1}}^{\sigma(s)} \frac{\Delta v}{r(v)}}-(r(s) a(s))^{\Delta}\right]+\frac{B^{2}(s)}{4 A(s)} \\
& -\frac{p_{0}}{\tau_{0}}\left[\eta^{\sigma}(s)\left[\tau_{0} r(\tau(s)) a^{2}(s) \frac{\int_{t_{1}}^{\tau(s)} \frac{\Delta v}{r(v)}}{\int_{t_{1}}^{\tau^{\sigma}(s)} \frac{\Delta v}{r(v)}}-(r(\tau(s)) a(s))^{\Delta}\right]-\frac{D^{2}(s)}{4 C(s)}\right], \\
A(s):= & \frac{\eta^{\sigma}(s)}{r(s) \eta^{2}(s)} \frac{\int_{t_{1}}^{s} \frac{\Delta v}{r(v)}}{\int_{t_{1}}^{\sigma(s)} \frac{\Delta v}{r(v)}}, \quad B(s):=\frac{\eta^{\Delta}(s)}{\eta(s)}+\frac{2 \eta^{\sigma}(s) a(s)}{\eta(s)} \frac{\int_{t_{1}}^{s} \frac{\Delta v}{r(v)}}{\int_{t_{1}}^{\sigma(s)} \frac{\Delta v}{r(v)}}, \\
C(s):= & \frac{\tau_{0} \eta^{\sigma}(s)}{r(\tau(s)) \eta^{2}(s)} \frac{\int_{t_{1}}^{\tau(s)} \frac{\Delta v}{r(v)}}{\int_{t_{1}}^{\tau^{\sigma}(s)} \frac{\Delta v}{r(v)}}, \quad D(s):=\frac{\eta^{\Delta}(s)}{\eta(s)}+\frac{2 \tau_{0} \eta^{\sigma}(s) a(s)}{\eta(s)} \frac{\int_{t_{1}}^{\tau(s)} \frac{\Delta v}{\tau^{\tau}(v)}}{\int_{t_{1}}^{\tau^{\sigma}(s)} \frac{\Delta v}{r(v)}},
\end{aligned}
$$

then (1.1) is oscillatory.

Proof Assume that (1.1) has a nonoscillatory solution $x$ on $\left[t_{0}, \infty\right)_{\mathbb{T}}$. Without loss of generality, suppose that it is an eventually positive solution. From (1.1) and [1, Theorem 1.93], we obtain

$$
\frac{p_{0}}{\tau^{\Delta}(t)}\left(r(\tau(t)) z^{\Delta}(\tau(t))\right)^{\Delta}+p_{0} q(\tau(t)) x(\delta(\tau(t)))=0 .
$$

Combining (1.1) and (2.2), we are led to

$$
\left(r(t) z^{\Delta}(t)\right)^{\Delta}+\frac{p_{0}}{\tau_{0}}\left(r(\tau(t)) z^{\Delta}(\tau(t))\right)^{\Delta}+Q(t) z(\delta(t)) \leq 0 .
$$

From (1.1) and (1.7), we have

$$
z^{\Delta}(t)>0 \quad \text { and } \quad\left(r(t) z^{\Delta}(t)\right)^{\Delta}<0
$$

for $t \in\left[t_{1}, \infty\right)_{\mathbb{T}}$, where $t_{1} \in\left[t_{0}, \infty\right)_{\mathbb{T}}$ is large enough. Define the function $\omega$ by

$$
\omega(t):=\eta(t)\left[\frac{r(t) z^{\Delta}(t)}{z(t)}+r(t) a(t)\right], \quad t \geq t_{1} .
$$

Hence, we have $\omega(t)>0$ for $t \in\left[t_{1}, \infty\right)_{\mathbb{T}}$ and

$$
\omega^{\Delta}=\eta^{\Delta}\left[\frac{r z^{\Delta}}{z}+r a\right]+\eta^{\sigma}\left[\frac{r z^{\Delta}}{z}+r a\right]^{\Delta}
$$




$$
\begin{aligned}
& =\frac{\eta^{\Delta}}{\eta} \omega+\eta^{\sigma}(r a)^{\Delta}+\eta^{\sigma}\left[\frac{r z^{\Delta}}{z}\right]^{\Delta} \\
& =\frac{\eta^{\Delta}}{\eta} \omega+\eta^{\sigma}(r a)^{\Delta}+\eta^{\sigma} \frac{\left(r z^{\Delta}\right)^{\Delta} z-r\left(z^{\Delta}\right)^{2}}{z z^{\sigma}} \\
& =\frac{\eta^{\Delta}}{\eta} \omega+\eta^{\sigma}(r a)^{\Delta}+\eta^{\sigma} \frac{\left(r z^{\Delta}\right)^{\Delta}}{z^{\sigma}}-\eta^{\sigma} \frac{r\left(z^{\Delta}\right)^{2}}{z z^{\sigma}} \\
& =\frac{\eta^{\Delta}}{\eta} \omega+\eta^{\sigma}(r a)^{\Delta}+\eta^{\sigma} \frac{\left(r z^{\Delta}\right)^{\Delta}}{z^{\sigma}}-\eta^{\sigma} r\left(\frac{z^{\Delta}}{z}\right)^{2} \frac{z}{z^{\sigma}} .
\end{aligned}
$$

By virtue of $\left(r(t) z^{\Delta}(t)\right)^{\Delta}<0$, we have

$$
z(t) \geq z^{\Delta}(t) r(t) \int_{t_{1}}^{t} \frac{\Delta s}{r(s)}
$$

and so

$$
\left(\frac{z(t)}{\int_{t_{1}}^{t} \frac{\Delta s}{r(s)}}\right)^{\Delta} \leq 0
$$

which implies that

$$
\frac{z(t)}{z^{\sigma}(t)} \geq \frac{\int_{t_{1}}^{t} \frac{\Delta s}{r(s)}}{\int_{t_{1}}^{\sigma(t)} \frac{\Delta s}{r(s)}}
$$

On the other hand, we have by (2.4) that

$$
\left(\frac{z^{\Delta}}{z}\right)^{2}=\left[\frac{\omega}{r \eta}-a\right]^{2}=\left[\frac{\omega}{r \eta}\right]^{2}+a^{2}-2 \frac{\omega a}{r \eta} .
$$

Putting (2.7) and (2.8) into (2.5), we have

$$
\begin{aligned}
\omega^{\Delta} \leq & \eta^{\sigma} \frac{\left(r z^{\Delta}\right)^{\Delta}}{z^{\sigma}}-\eta^{\sigma}\left[r a^{2} \frac{\int_{t_{1}}^{t} \frac{\Delta s}{r(s)}}{\int_{t_{1}}^{\sigma(t)} \frac{\Delta s}{r(s)}}-(r a)^{\Delta}\right] \\
& +\left[\frac{\eta^{\Delta}}{\eta}+\frac{2 \eta^{\sigma} a}{\eta} \frac{\int_{t_{1}}^{t} \frac{\Delta s}{r(s)}}{\int_{t_{1}}^{\sigma(t)} \frac{\Delta s}{r(s)}}\right] \omega-\frac{\eta^{\sigma}}{r \eta^{2}} \frac{\int_{t_{1}}^{t} \frac{\Delta s}{r(s)}}{\int_{t_{1}}^{\sigma(t)} \frac{\Delta s}{r(s)}} \omega^{2} \\
\leq & \eta^{\sigma} \frac{\left(r z^{\Delta}\right)^{\Delta}}{z^{\sigma}}-\eta^{\sigma}\left[r a^{2} \frac{\int_{t_{1}}^{t} \frac{\Delta s}{r(s)}}{\int_{t_{1}}^{\sigma(t)} \frac{\Delta s}{r(s)}}-(r a)^{\Delta}\right]+\frac{B^{2}}{4 A}
\end{aligned}
$$

Now, define the function $u$ by

$$
u(t):=\eta(t)\left[\frac{r(\tau(t)) z^{\Delta}(\tau(t))}{z(\tau(t))}+r(\tau(t)) a(t)\right], \quad t \geq t_{1}
$$

Hence, we have by [1, Theorem 1.93$]$ and $\left(H_{3}\right)$ that

$$
u^{\Delta}=\eta^{\Delta}\left[\frac{(r \circ \tau)\left(z^{\Delta} \circ \tau\right)}{z \circ \tau}+(r \circ \tau) a\right]+\eta^{\sigma}\left[\frac{(r \circ \tau)\left(z^{\Delta} \circ \tau\right)}{z \circ \tau}+(r \circ \tau) a\right]^{\Delta}
$$




$$
\begin{aligned}
= & \frac{\eta^{\Delta}}{\eta} u+\eta^{\sigma}((r \circ \tau) a)^{\Delta}+\eta^{\sigma}\left[\frac{(r \circ \tau)\left(z^{\Delta} \circ \tau\right)}{z \circ \tau}\right]^{\Delta} \\
= & \frac{\eta^{\Delta}}{\eta} u+\eta^{\sigma}((r \circ \tau) a)^{\Delta}+\eta^{\sigma} \frac{\left((r \circ \tau)\left(z^{\Delta} \circ \tau\right)\right)^{\Delta}(z \circ \tau)-(r \circ \tau)\left(z^{\Delta} \circ \tau\right)\left(z^{\Delta} \circ \tau\right) \tau^{\Delta}}{(z \circ \tau)\left(z \circ \tau^{\sigma}\right)} \\
= & \frac{\eta^{\Delta}}{\eta} u+\eta^{\sigma}((r \circ \tau) a)^{\Delta}+\eta^{\sigma} \frac{\left((r \circ \tau)\left(z^{\Delta} \circ \tau\right)\right)^{\Delta}}{z \circ \tau^{\sigma}}-\eta^{\sigma} \tau^{\Delta}(r \circ \tau)\left(\frac{z^{\Delta} \circ \tau}{z \circ \tau}\right)^{2} \frac{z \circ \tau}{z \circ \tau^{\sigma}} \\
\leq & \frac{\eta^{\Delta}}{\eta} u+\eta^{\sigma}((r \circ \tau) a)^{\Delta}+\eta^{\sigma} \frac{\left((r \circ \tau)\left(z^{\Delta} \circ \tau\right)\right)^{\Delta}}{z \circ \tau^{\sigma}} \\
& -\tau_{0} \eta^{\sigma}(r \circ \tau)\left(\frac{z^{\Delta} \circ \tau}{z \circ \tau}\right)^{2} \frac{z \circ \tau}{z \circ \tau^{\sigma}} .
\end{aligned}
$$

Note that (2.6) implies that

$$
\frac{z(\tau(t))}{z\left(\tau^{\sigma}(t)\right)} \geq \frac{\int_{t_{1}}^{\tau(t)} \frac{\Delta s}{r(s)}}{\int_{t_{1}}^{\tau^{\sigma}(t)} \frac{\Delta s}{r(s)}} .
$$

On the other hand, we have by (2.10) that

$$
\left(\frac{z^{\Delta} \circ \tau}{z \circ \tau}\right)^{2}=\left[\frac{u}{(r \circ \tau) \eta}-a\right]^{2}=\left[\frac{u}{(r \circ \tau) \eta}\right]^{2}+a^{2}-2 \frac{u a}{(r \circ \tau) \eta} .
$$

Putting (2.12) and (2.13) into (2.11), we have

$$
\begin{aligned}
u^{\Delta} \leq & \eta^{\sigma} \frac{\left((r \circ \tau)\left(z^{\Delta} \circ \tau\right)\right)^{\Delta}}{z \circ \tau^{\sigma}}-\eta^{\sigma}\left[\tau_{0}(r \circ \tau) a^{2} \frac{\int_{t_{1}}^{\tau(t)} \frac{\Delta s}{r(s)}}{\int_{t_{1}}^{\tau^{\sigma}(t)} \frac{\Delta s}{r(s)}}-((r \circ \tau) a)^{\Delta}\right] \\
& +\left[\frac{\eta^{\Delta}}{\eta}+\frac{2 \tau_{0} \eta^{\sigma} a}{\eta} \frac{\int_{t_{1}(t)}^{\tau(t)} \frac{\Delta s}{r(s)}}{\int_{t_{1}}^{\tau^{\sigma}(t)} \frac{\Delta s}{r(s)}}\right] u-\frac{\tau_{0} \eta^{\sigma}}{(r \circ \tau) \eta^{2}} \frac{\int_{t_{1}}^{\tau(t)} \frac{\Delta s}{\int_{t_{1}}^{\tau^{\sigma}(t)} \frac{\Delta s}{r(s)}} u^{2}}{\leq} \\
\leq & \eta^{\sigma} \frac{\left((r \circ \tau)\left(z^{\Delta} \circ \tau\right)\right)^{\Delta}}{z^{\sigma}} \frac{z^{\sigma}}{z \circ \tau^{\sigma}}-\eta^{\sigma}\left[\tau_{0}(r \circ \tau) a^{2} \frac{\int_{t_{1}}^{\tau(t)} \frac{\Delta s}{r(s)}}{\int_{t_{1}}^{\tau^{\sigma}(t)} \frac{\Delta s}{r(s)}}-((r \circ \tau) a)^{\Delta}\right]+\frac{D^{2}}{4 C} \\
\leq & \eta^{\sigma} \frac{\left((r \circ \tau)\left(z^{\Delta} \circ \tau\right)\right)^{\Delta}}{z^{\sigma}}-\eta^{\sigma}\left[\tau_{0}(r \circ \tau) a^{2} \frac{\int_{t_{1}}^{\tau(t)} \frac{\Delta s}{r(s)}}{\int_{t_{1}}^{\tau^{\sigma}(t)} \frac{\Delta s}{r(s)}}-((r \circ \tau) a)^{\Delta}\right]+\frac{D^{2}}{4 C} .
\end{aligned}
$$

Recalling (2.9) and (2.14), we have by (2.3) and (2.6) that

$$
\begin{aligned}
\omega^{\Delta}+\frac{p_{0}}{\tau_{0}} u^{\Delta} & \leq \eta^{\sigma} \frac{\left(r z^{\Delta}\right)^{\Delta}+\frac{p_{0}}{\tau_{0}}\left((r \circ \tau)\left(z^{\Delta} \circ \tau\right)\right)^{\Delta}}{z^{\sigma}}+E \\
& \leq-\eta^{\sigma} Q \frac{z \circ \delta}{z^{\sigma}}+E \\
& \leq-\eta^{\sigma} Q \frac{\int_{t_{1}}^{\delta(t)} \frac{\Delta s}{r(s)}}{\int_{t_{1}}^{\sigma(t)} \frac{\Delta s}{r(s)}}+E .
\end{aligned}
$$


Hence, we have

$$
\int_{t_{2}}^{t}\left(\eta^{\sigma}(s) Q(s) \frac{\int_{t_{1}}^{\delta(s)} \frac{\Delta v}{r(v)}}{\int_{t_{1}}^{\sigma(s)} \frac{\Delta v}{r(v)}}-E(s)\right) \Delta s \leq \omega\left(t_{2}\right)+\frac{p_{0}}{\tau_{0}} u\left(t_{2}\right)
$$

which contradicts (2.1). The proof is complete.

Based on Theorem 2.1, we have the following corollary when $\eta(t)=t$ and $a(t)=0$.

Corollary 2.2 Assume $\left(H_{1}\right)-\left(H_{3}\right)$ and (1.7). If

$$
\limsup _{t \rightarrow \infty} \int_{t_{2}}^{t}\left(\sigma(s) Q(s) \frac{\int_{t_{1}}^{\delta(s)} \frac{\Delta v}{r(v)}}{\int_{t_{1}}^{\sigma(s)} \frac{\Delta v}{r(v)}}-\frac{1}{4 \sigma(s)}\left(r(s) \frac{\int_{t_{1}}^{\sigma(s)} \frac{\Delta v}{r(v)}}{\int_{t_{1}}^{s} \frac{\Delta v}{r(v)}}+\frac{p_{0}}{\tau_{0}^{2}} r(\tau(s)) \frac{\int_{t_{1}}^{\tau^{\sigma}(s)} \frac{\Delta v}{r(v)}}{\int_{t_{1}}^{\tau(s)} \frac{\Delta v}{r(v)}}\right)\right) \Delta s=\infty
$$

for all sufficiently large $t_{1}$ and for some $t_{2} \geq t_{1}$, then (1.1) is oscillatory.

When $\mathbb{T}=\mathbb{R}$, we have from Corollary 2.2 the following result for the neutral differential equation (1.3).

Corollary 2.3 Assume $\left(H_{1}\right)-\left(H_{3}\right)$ for $\mathbb{T}=\mathbb{R}$ and (1.2). If

$$
\limsup _{t \rightarrow \infty} \int_{t_{2}}^{t}\left(s Q(s) \frac{\int_{t_{1}}^{\delta(s)} \frac{\mathrm{d} v}{r(v)}}{\int_{t_{1}}^{s} \frac{\mathrm{d} v}{r(v)}}-\frac{1}{4 s}\left(r(s)+\frac{p_{0}}{\tau_{0}^{2}} r(\tau(s))\right)\right) \mathrm{d} s=\infty
$$

for all sufficiently large $t_{1}$ and for some $t_{2} \geq t_{1}$, then (1.3) is oscillatory.

Example 2.4 Consider the second-order neutral differential equation

$$
\left(x(t)+\frac{1}{2} x(t-1)\right)^{\prime \prime}+\frac{\gamma}{t^{2}} x(t)=0 \quad \text { for } t \geq 1
$$

where $\gamma>0$ is a constant, $r(t)=1, p_{0}=1 / 2, \tau_{0}=1, q(t)=Q(t)=\gamma / t^{2}, \tau(t)=t-1$, and $\delta(t)=t$. Note that

$$
\begin{gathered}
\limsup _{t \rightarrow \infty} \int_{t_{2}}^{t}\left(s Q(s) \frac{\int_{t_{1}}^{\delta(s)} \frac{\mathrm{d} v}{r(v)}}{\int_{t_{1}}^{s} \frac{\mathrm{d} v}{r(v)}}-\frac{1}{4 s}\left(r(s)+\frac{p_{0}}{\tau_{0}^{2}} r(\tau(s))\right)\right) \mathrm{d} s \\
\quad=\left[\gamma-\frac{3}{8}\right] \limsup _{t \rightarrow \infty} \int_{t_{2}}^{t} \frac{\mathrm{d} s}{s}=\infty, \quad \text { if } \gamma>\frac{3}{8} .
\end{gathered}
$$

Hence, by Corollary 2.3, (2.15) is oscillatory if $\gamma>3 / 8$. Let $\theta(t)=t$ and $p=p(t)=1 / 2$. Then

$$
\int_{t_{0}}^{\infty}\left(\theta(s) q(s)[1-p(s-\delta)]-\frac{\left(\theta^{\prime}(s)\right)^{2} r(s-\delta)}{4 \theta(s)}\right) \mathrm{d} s=\infty, \quad \text { if } \gamma>1 / 2
$$

and

$$
\int_{t_{0}}^{\infty}\left(\theta(s) q(s) \frac{1-\varepsilon}{1+p(1+\varepsilon)}-\frac{\left(\theta^{\prime}(s)\right)^{2} r(\delta(s))}{4 \theta(s) \delta^{\prime}(s)}\right) \mathrm{d} s=\infty, \quad \text { if } \gamma>\frac{1+\frac{1}{2}(1+\varepsilon)}{4(1-\varepsilon)}
$$


for some constant $\varepsilon \in(0,1)$. Since

$$
\frac{1+\frac{1}{2}(1+\varepsilon)}{4(1-\varepsilon)}>\frac{3}{8}
$$

our result is better than $[15,38]$ in some cases.

Example 2.5 For $t \geq 1$, consider the second-order neutral delay differential equation

$$
\left(t^{1 / 2}\left[x(t)+p_{0} x\left(\frac{t}{2}\right)\right]^{\prime}\right)^{\prime}+\frac{a}{t^{3 / 2}} x\left(\frac{t}{4}\right)=0
$$

where $0<p_{0}<\infty$ and $a>0$ is a constant. In [8], Baculíková and Džurina obtained that the condition

$$
a>\frac{1+2 p_{0}}{\mathrm{e} \ln 2}
$$

ensures oscillation of (2.16) (using Theorem 1.1). Letting $\eta(t)=t^{\frac{1}{2}}$, an application of Theorem 2.1 yields that the condition

$$
a>\frac{1}{k_{0}}\left(\frac{1}{8}+\frac{\sqrt{2} p_{0}}{4}\right) \text { for some constant } k_{0} \in(0,1)
$$

guarantees oscillation of (2.16). For example, we can put $a>1 / 6+\sqrt{2} p_{0} / 3$ (by letting $k_{0}=$ $3 / 4)$. Hence, our result improves that in [8] since

$$
\frac{1+2 p_{0}}{\mathrm{e} \ln 2}>\frac{1}{6}+\frac{\sqrt{2} p_{0}}{3}
$$

Example 2.6 For $t \geq 1$, consider the second-order neutral delay differential equation

$$
\left[x(t)+p_{0} x\left(\frac{t}{2}\right)\right]^{\prime \prime}+\frac{a}{t^{2}} x(t)=0,
$$

where $0<p_{0}<\infty$ and $a>0$ is a constant. An application of Corollary 2.3 implies that the condition

$$
a>\frac{1}{4}+p_{0}
$$

guarantees oscillation of (2.17). However, applications of Theorem 1.2 and Theorem 1.4 (by letting $\alpha(t)=t$ ) yield that

$$
a>\frac{1}{2}+p_{0}
$$

ensures oscillation of (2.17). Hence, our result is new. Note that Theorem 1.3 and Theorem 1.5 cannot be applied in (2.17). 
In the following, we give an oscillation criterion for (1.1) when

$$
\int_{t_{0}}^{\infty} \frac{\Delta t}{r(t)}<\infty
$$

Theorem 2.7 Assume $\left(H_{1}\right)-\left(H_{3}\right)$ and (2.18). Suppose further that there exist two functions $\eta, a \in \mathrm{C}_{\mathrm{rd}}^{1}\left(\left[t_{0}, \infty\right)_{\mathbb{T}}, \mathbb{R}\right)$ such that $\eta(t)>0, a(t) \geq 0$, and (2.1) holds for all sufficiently large $t_{1}$ and for some $t_{2} \geq t_{1}$. If there exists a positive function $b \in \mathrm{C}_{\mathrm{rd}}^{1}\left(\left[t_{0}, \infty\right)_{\mathbb{T}}, \mathbb{R}\right)$ such that

$$
b(t)-\frac{1}{r(t) R(t)} \geq 0, \quad b(t)-\frac{1}{r(\tau(t)) R(\tau(t))} \geq 0,
$$

and

$$
\limsup _{t \rightarrow \infty} \int_{t_{0}}^{t}\left(\eta^{\sigma}(s) Q(s) \frac{R^{\sigma}(s)}{R\left(\tau^{\sigma}(s)\right)}-E_{*}(s)\right) \Delta s=\infty,
$$

where

$$
\begin{aligned}
R(t):= & \int_{t}^{\infty} \frac{\Delta s}{r(s)}, \\
E_{*}(s):= & -\eta^{\sigma}(s)\left[r(s) b^{2}(s)-(r(s) b(s))^{\Delta}\right]+\frac{B_{*}^{2}(s)}{4 A_{*}(s)} \\
& -\frac{p_{0}}{\tau_{0}}\left[\eta^{\sigma}(s)\left[\tau_{0} r(\tau(s)) b^{2}(s)-(r(\tau(s)) b(s))^{\Delta}\right]-\frac{D_{*}^{2}(s)}{4 C_{*}(s)}\right], \\
A_{*}(s):= & \frac{\eta^{\sigma}(s)}{r(s) \eta^{2}(s)}, \quad B *(s):=\frac{\eta^{\Delta}(s)}{\eta(s)}+\frac{2 \eta^{\sigma}(s) b(s)}{\eta(s)}, \\
C_{*}(s):= & \frac{\tau_{0} \eta^{\sigma}(s)}{r(\tau(s)) \eta^{2}(s)}, \quad D_{*}(s):=\frac{\eta^{\Delta}(s)}{\eta(s)}+\frac{2 \tau_{0} \eta^{\sigma}(s) b(s)}{\eta(s)},
\end{aligned}
$$

then (1.1) is oscillatory.

Proof Assume that (1.1) has a nonoscillatory solution $x$ on $\left[t_{0}, \infty\right)_{\mathbb{T}}$. Without loss of generality, suppose that it is an eventually positive solution. By the proof of Theorem 2.1, we have (2.3). From (1.1), there exists $t_{1} \in\left[t_{0}, \infty\right)_{\mathbb{T}}$ such that $z^{\Delta}(t)>0$ or $z^{\Delta}(t)<0$ for $t \in\left[t_{1}, \infty\right)_{\mathbb{T}}$. The proof of the case when $z^{\Delta}(t)>0$ is the same as that of Theorem 2.1, and we can get a contradiction to (2.1). Now, we assume $z^{\Delta}(t)<0$. Then we have

$$
z^{\Delta}(s) \leq \frac{r(t)}{r(s)} z^{\Delta}(t), \quad s \geq t \geq t_{1}
$$

Integrating this from $t$ to $\infty$, we get

$$
\frac{z^{\Delta}(t)}{z(t)} \geq-\frac{1}{r(t) R(t)}, \quad \frac{z^{\Delta}(\tau(t))}{z(\tau(t))} \geq-\frac{1}{r(\tau(t)) R(\tau(t))},
$$

and

$$
\left(\frac{z}{R}\right)^{\Delta} \geq 0
$$


Define the function $\omega$ by

$$
\omega(t):=\eta(t)\left[\frac{r(t) z^{\Delta}(t)}{z(t)}+r(t) b(t)\right], \quad t \geq t_{1} .
$$

Then $\omega(t) \geq 0$,

$$
\omega^{\Delta}=\frac{\eta^{\Delta}}{\eta} \omega+\eta^{\sigma}(r b)^{\Delta}+\eta^{\sigma} \frac{\left(r z^{\Delta}\right)^{\Delta}}{z^{\sigma}}-\eta^{\sigma} r\left(\frac{z^{\Delta}}{z}\right)^{2} \frac{z}{z^{\sigma}},
$$

and

$$
\left(\frac{z^{\Delta}}{z}\right)^{2}=\left[\frac{\omega}{r \eta}-b\right]^{2}=\left[\frac{\omega}{r \eta}\right]^{2}+b^{2}-2 \frac{\omega b}{r \eta} .
$$

Note that $z / z^{\sigma} \geq 1$. By virtue of (2.22) and (2.23), we have

$$
\begin{aligned}
\omega^{\Delta} & \leq \eta^{\sigma} \frac{\left(r z^{\Delta}\right)^{\Delta}}{z^{\sigma}}-\eta^{\sigma}\left[r b^{2}-(r b)^{\Delta}\right]+\left[\frac{\eta^{\Delta}}{\eta}+\frac{2 \eta^{\sigma} b}{\eta}\right] \omega-\frac{\eta^{\sigma}}{r \eta^{2}} \omega^{2} \\
& \leq \eta^{\sigma} \frac{\left(r z^{\Delta}\right)^{\Delta}}{z \circ \tau^{\sigma}}-\eta^{\sigma}\left[r b^{2}-(r b)^{\Delta}\right]+\frac{B_{*}^{2}}{4 A_{*}^{*}} .
\end{aligned}
$$

Now, define the function $u$ by

$$
u(t):=\eta(t)\left[\frac{r(\tau(t)) z^{\Delta}(\tau(t))}{z(\tau(t))}+r(\tau(t)) b(t)\right], \quad t \geq t_{1} .
$$

Hence, we have $u(t) \geq 0$,

$$
\begin{aligned}
u^{\Delta} \leq & \frac{\eta^{\Delta}}{\eta} u+\eta^{\sigma}((r \circ \tau) b)^{\Delta}+\eta^{\sigma} \frac{\left((r \circ \tau)\left(z^{\Delta} \circ \tau\right)\right)^{\Delta}}{z \circ \tau^{\sigma}} \\
& -\tau_{0} \eta^{\sigma}(r \circ \tau)\left(\frac{z^{\Delta} \circ \tau}{z \circ \tau}\right)^{2} \frac{z \circ \tau}{z \circ \tau^{\sigma}}
\end{aligned}
$$

and

$$
\left(\frac{z^{\Delta} \circ \tau}{z \circ \tau}\right)^{2}=\left[\frac{u}{(r \circ \tau) \eta}-b\right]^{2}=\left[\frac{u}{(r \circ \tau) \eta}\right]^{2}+b^{2}-2 \frac{u b}{(r \circ \tau) \eta} .
$$

Note that $z(\tau(t)) / z\left(\tau^{\sigma}(t)\right) \geq 1$. By virtue of (2.25) and (2.26), we have

$$
\begin{aligned}
u^{\Delta} \leq & \eta^{\sigma} \frac{\left((r \circ \tau)\left(z^{\Delta} \circ \tau\right)\right)^{\Delta}}{z \circ \tau^{\sigma}}-\eta^{\sigma}\left[\tau_{0}(r \circ \tau) b^{2}-((r \circ \tau) b)^{\Delta}\right] \\
& +\left[\frac{\eta^{\Delta}}{\eta}+\frac{2 \tau_{0} \eta^{\sigma} b}{\eta}\right] u-\frac{\tau_{0} \eta^{\sigma}}{(r \circ \tau) \eta^{2}} u^{2} \\
\leq & \eta^{\sigma} \frac{\left((r \circ \tau)\left(z^{\Delta} \circ \tau\right)\right)^{\Delta}}{z \circ \tau^{\sigma}}-\eta^{\sigma}\left[\tau_{0}(r \circ \tau) b^{2}-((r \circ \tau) b)^{\Delta}\right]+\frac{D_{*}^{2}}{4 C_{*}} .
\end{aligned}
$$

Recalling (2.24) and (2.27), we have by (2.3) and (2.21) that

$$
\omega^{\Delta}+\frac{p_{0}}{\tau_{0}} u^{\Delta} \leq \eta^{\sigma} \frac{\left(r z^{\Delta}\right)^{\Delta}+\frac{p_{0}}{\tau_{0}}\left((r \circ \tau)\left(z^{\Delta} \circ \tau\right)\right)^{\Delta}}{z^{\sigma}} \frac{z^{\sigma}}{z \circ \tau^{\sigma}}+E^{*} \leq-\eta^{\sigma} Q \frac{R^{\sigma}}{R \circ \tau^{\sigma}}+E_{*} .
$$


Hence, we have

$$
\int_{t_{1}}^{t}\left(\eta^{\sigma}(s) Q(s) \frac{R^{\sigma}(s)}{R\left(\tau^{\sigma}(s)\right)}-E_{*}(s)\right) \Delta s \leq \omega\left(t_{1}\right)+\frac{p_{0}}{\tau_{0}} u\left(t_{1}\right),
$$

which contradicts (2.20). The proof is complete.

Example 2.8 Consider the second-order neutral differential equation

$$
\left(t^{2}\left(x(t)+p_{0} x(t-1)\right)^{\prime}\right)^{\prime}+q_{0} x(t)=0 \quad \text { for } t \geq 2,
$$

where $r(t)=t^{2}, \tau_{0}=1, q(t)=Q(t)=q_{0}, \tau(t)=t-1$, and $\delta(t)=t$. Note that $R(t)=t^{-1}$. Let $\eta(t)=1 / t$ and $a(t)=b(t)=1 /(t-1)$. Then

$$
\begin{aligned}
& \limsup _{t \rightarrow \infty} \int_{t_{2}}^{t}\left(\eta^{\sigma}(s) Q(s) \frac{\int_{t_{1}}^{\delta(s)} \frac{\Delta v}{r(v)}}{\int_{t_{1}}^{\sigma(s)} \frac{\Delta v}{r(v)}}-E(s)\right) \Delta s \\
& \quad=\limsup _{t \rightarrow \infty} \int_{t_{2}}^{t}\left[\frac{q_{0}}{s}+\frac{2}{(s-1)^{2}}-\frac{(s+1)^{2}}{4 s(s-1)^{2}}-p_{0} \frac{(s+1)^{2}}{4 s^{3}}\right] \mathrm{d} s \\
& =\infty, \quad \text { if } q_{0}>\frac{1+p_{0}}{4}
\end{aligned}
$$

and

$$
\begin{aligned}
& \limsup _{t \rightarrow \infty} \int_{t_{0}}^{t}\left(\eta^{\sigma}(s) Q(s) \frac{R^{\sigma}(s)}{R\left(\tau^{\sigma}(s)\right)}-E_{*}(s)\right) \Delta s \\
& \quad=\limsup _{t \rightarrow \infty} \int_{2}^{t}\left[\frac{q_{0}(s-1)}{s^{2}}+\frac{2}{(s-1)^{2}}-\frac{(s+1)^{2}}{4 s(s-1)^{2}}-p_{0} \frac{(s+1)^{2}}{4 s^{3}}\right] \mathrm{d} s \\
& \quad=\infty, \quad \text { if } q_{0}>\frac{1+p_{0}}{4} .
\end{aligned}
$$

Hence, by Theorem 2.7, (2.28) is oscillatory if $q_{0}>\left(1+p_{0}\right) / 4$. When $p_{0}=0, q_{0}>1 / 4$ is a sharp condition for oscillation of the equation $\left(t^{2} x^{\prime}(t)\right)^{\prime}+q_{0} x(t)=0$. Note that the results of $[13,36]$ cannot give this result (see (1.5)), and hence our results improve those of [13, 36].

\section{Discussions}

In this paper, we have suggested some new oscillation criteria for second-order neutral delay dynamic equation (1.1) by employing the generalized Riccati substitution. To achieve these results, we are forced to require, similar as in [33], that $\tau^{\Delta}(t) \geq \tau_{0}>0, \tau \circ \delta=\delta \circ \tau$, and $\tau\left(\left[t_{0}, \infty\right)_{\mathbb{T}}\right)=\left[\tau\left(t_{0}\right), \infty\right)_{\mathbb{T}}$. It would be interesting to seek other methods for further study of oscillatory properties or asymptotic problems of equation (1.1) in the case where $p(t) \geq 1$.

During the past three decades, there have been many classical results regarding oscillatory behavior of equation (1.1) in the case where $\mathbb{T}=\mathbb{R}$, some of which provided that $0 \leq p(t)<1$; see, for example, $[15,16]$. Examples given in this paper reveal some advantages even when one applies the obtained criteria to the case where $0 \leq p(t)<1$. 
These results show that the delay argument $\tau$ plays an important role in oscillation of second-order neutral delay dynamic equations; see the details in Example 2.6 and differences between Corollary 2.3 and Theorem 1.2, Theorem 1.4. Let us go through Example 2.6. One can easily see some superiorities in comparison to those related results, e.g., Theorem 1.2 and Theorem 1.4.

As a special case when $p(t)=0$, the established results improve those of $[13,36]$ in some sense, which is shown by Example 2.8.

\section{Competing interests}

The authors declare that they have no competing interests.

\section{Authors' contributions}

All authors contributed equally to the writing of the paper. All authors read and approved the final manuscript.

\section{Author details}

${ }^{1}$ School of Control Science and Engineering, Shandong University, Jinan, Shandong 250061, P.R. China. ${ }^{2}$ Department of Mathematics, Texas A\&M University-Kingsville, 700 University Blvd., Kingsville, TX 78363-8202, USA. ${ }^{3}$ Department of Mathematics and Statistics, Missouri S\&T, Rolla, MO 65409-0020, USA.

\section{Acknowledgements}

This research is supported by NNSF of P.R. China (Grant Nos. 61034007, 51277116, 50977054).

Received: 9 October 2012 Accepted: 3 December 2012 Published: 28 December 2012

\section{References}

1. Bohner, M, Peterson, A: Dynamic Equations on Time Scales: an Introduction with Applications. Birkhäuser, Boston (2001)

2. Bohner, M, Peterson, A: Advances in Dynamic Equations on Time Scales. Birkhäuser, Boston (2003)

3. Agarwal, RP, Bohner, M, Tang, S, Li, T, Zhang, C: Oscillation and asymptotic behavior of third-order nonlinear retarded dynamic equations. Appl. Math. Comput. 219(8), 3600-3609 (2012)

4. Agarwal, RP, O'Regan, D, Saker, SH: Oscillation criteria for second-order nonlinear neutral delay dynamic equations. J. Math. Anal. Appl. 300, 203-217 (2004)

5. Agarwal, RP, O'Regan, D, Saker, SH: Oscillation results for second-order nonlinear neutral delay dynamic equations on time scales. Appl. Anal. 86, 1-17 (2007)

6. Agarwal, RP, O'Regan, D, Saker, SH: Oscillation theorems for second-order nonlinear neutral delay dynamic equations on time scales. Acta Math. Sin. 24, 1409-1432 (2008)

7. Akın-Bohner, E, Bohner, M, Saker, SH: Oscillation criteria for a certain class of second order Emden-Fowler dynamic equations. Electron. Trans. Numer. Anal. 27, 1-12 (2007)

8. Baculíková, B, Džurina, J: Oscillation theorems for second order neutral differential equations. Comput. Math. Appl. 61, 94-99 (2011)

9. Bohner, M, Saker, SH: Oscillation of second order nonlinear dynamic equations on time scales. Rocky Mt. J. Math. 34, 1239-1254 (2004)

10. Bohner, M, Saker, SH: Oscillation criteria for perturbed nonlinear dynamic equations. Math. Comput. Model. 40, 249-260 (2004)

11. Erbe, L, Peterson, A, Saker, SH: Oscillation criteria for second-order nonlinear dynamic equations on time scales. J. Lond. Math. Soc. 67, 701-714 (2003)

12. Erbe, L, Peterson, A, Saker, SH: Kamenev-type oscillation criteria for second-order linear delay dynamic equations. Dyn. Syst. Appl. 15, 65-78 (2006)

13. Erbe, L, Peterson, A, Saker, SH: Oscillation criteria for second-order nonlinear delay dynamic equations. J. Math. Anal. Appl. 333, 505-522 (2007)

14. Grace, SR, Bohner, M, Agarwal, RP: On the oscillation of second-order half-linear dynamic equations. J. Differ. Equ. Appl. 15, 451-460 (2009)

15. Grace, SR, Lalli, BS: Oscillation of nonlinear second order neutral delay differential equations. Rad. Mat. 3, 77-84 (1987)

16. Grammatikopoulos, MK, Ladas, G, Meimaridou, A: Oscillation of second order neutral delay differential equation. Rad. Mat. 1, 267-274 (1985)

17. Han, Z, Li, T, Sun, S, Sun, Y: Remarks on the paper [Appl. Math. Comput. 207 (2009) 388-396]. Appl. Math. Comput. 215, 3998-4007 (2010)

18. Han, Z, Li, T, Sun, S, Zhang, C: Oscillation for second-order nonlinear delay dynamic equations on time scales. Adv. Differ. Equ. 2009, 1-13 (2009)

19. Han, Z, Li, T, Sun, S, Zhang, C: On the oscillation of second-order neutral delay dynamic equations on time scales. African Diaspora J. Math. 9, 76-86 (2010)

20. Hasanbulli, M, Rogovchenko, YV: Oscillation criteria for second-order nonlinear neutral differential equations. Appl. Math. Comput. 215, 4392-4399 (2010)

21. Higgins, R: Asymptotic behavior of second-order nonlinear dynamic equations on time scales. Discrete Contin. Dyn. Syst., Ser. B 13, 609-622 (2010)

22. Karpuz, B: Asymptotic behavior of bounded solutions of a class of higher-order neutral dynamic equations. Appl. Math. Comput. 215, 2174-2183 (2009) 
23. Li, T, Agarwal, RP, Bohner, M: Some oscillation results for second-order neutral dynamic equations, Hacet. J. Math. Stat. 41(5), 715-721 (2012)

24. Li, T, Han, Z, Sun, S, Yang, D: Existence of nonoscillatory solutions to second-order neutral delay dynamic equations on time scales. Adv. Differ. Equ. 2009, 1-10 (2009)

25. Li, T, Thandapani, E: Oscillation of second-order quasi-linear neutral functional dynamic equations with distributed deviating arguments. J. Nonlinear Sci. Appl. 4, 180-192 (2011)

26. Şahiner, Y: Oscillation of second-order delay differential equations on time scales. Nonlinear Anal. 63, 1073-1080 (2005)

27. Şahiner, Y: Oscillation of second-order neutral delay and mixed-type dynamic equations on time scales. Adv. Differ. Equ. 2006, 1-9 (2006)

28. Saker, SH: Oscillation of nonlinear dynamic equations on time scales. Appl. Math. Comput. 148, 81-91 (2004)

29. Saker, SH: Oscillation of second-order nonlinear neutral delay dynamic equations on time scales. J. Comput. Appl. Math. 177, 375-387 (2005)

30. Saker, SH: Oscillation of second-order neutral delay dynamic equations of Emden-Fowler type. Dyn. Syst. Appl. 15 629-644 (2006)

31. Saker, SH, O'Regan, D: New oscillation criteria for second-order neutral functional dynamic equations via the generalized Riccati substitution. Commun. Nonlinear Sci. Numer. Simul. 16, 423-434 (2011)

32. Sun, S, Li, T, Han, Z, Zhang, C: On oscillation of second-order nonlinear neutral functional differential equations. Bull. Malays. Math. Sci. Soc. (2012, in press)

33. Tripathy, AK: Some oscillation results for second-order nonlinear dynamic equations of neutral type. Nonlinear Anal. 71, 727-735 (2009)

34. Zhang, BG, Shanliang, Z: Oscillation of second-order nonlinear delay dynamic equations on time scales. Comput. Math. Appl. 49, 599-609 (2005)

35. Zhang, C, Li, T, Agarwal, RP, Bohner, M: Oscillation results for fourth-order nonlinear dynamic equations. Appl. Math. Lett. 25, 2058-2065 (2012)

36. Zhang, Q: Oscillation of second-order half-linear delay dynamic equations with damping on time scales. J. Comput. Appl. Math. 235, 1180-1188 (2011)

37. Zhang, S, Wang, Q: Oscillation of second-order nonlinear neutral dynamic equations on time scales. Appl. Math. Comput. 216, 2837-2848 (2010)

38. Zhong, J, Ouyang, Z, Zou, S: An oscillation theorem for a class of second-order forced neutral delay differential equations with mixed nonlinearities. Appl. Math. Lett. 24, 1449-1454 (2011)

doi:10.1186/1687-1847-2012-227

Cite this article as: Zhang et al.: New oscillation results for second-order neutral delay dynamic equations. Advances in Difference Equations 2012 2012:227.

\section{Submit your manuscript to a SpringerOpen ${ }^{\circ}$ journal and benefit from:}

- Convenient online submission

- Rigorous peer review

- Immediate publication on acceptance

- Open access: articles freely available online

- High visibility within the field

- Retaining the copyright to your article 